\title{
Effets des défauts de soudure aluminothermique sur le comportement mécanique des rails
}

\author{
Ilias Mouallif ${ }^{a}$, AbDelkrim Chouaf, Abdelhamid El amri et Abdelkader Benali
}

Laboratoire de Contrôle et de Caractérisation Mécanique des Matériaux et des Structures (LCCMMS), Département de Génie Mécanique, École Nationale Supérieure d'Électricité et de Mécanique (ENSEM), Université Hassan II Casablanca, BP 8 118, Oasis, Route d'El Jadida, Casablanca, Maroc

Reçu le 27 octobre 2010, accepté le 7 juin 2011

\begin{abstract}
Résumé - L'assemblage des rails de chemin de fer se fait généralement à travers un procédé de soudage par aluminothermie. Ce procédé consiste en la coulée de métal de jonction entre les deux extrémités de rails à assembler. La qualité de cet assemblage dépend étroitement des conditions de la réalisation qui sont généralement difficiles à respecter. En effet, les techniques classiques de contrôle des rails, mettent souvent, en évidence la présence des défauts de soudure dans la jonction. Selon les observations, ces défauts peuvent être classés en deux types : défauts d'adhésion (ou défauts de collage) et défauts de porosité (ou défauts de soufflures multiples). Chacun de ces défauts, peut constituer un site privilégié de concentration des contraintes. Selon le niveau de ces contraintes, des éventuels processus d'endommagement risquent de se développer et mettant le rail hors service. Il semble donc nécessaire de modéliser le comportement mécanique de ces soudures avec ces défauts en vue de les classer par leur criticité. Dans cette étude, nous avons donc modélisé avec un même chargement et avec les mêmes conditions aux limites, la jonction de soudure pour chaque type de défaut. Les résultats obtenus sont très concluants et permettent de bien orienter l'approfondissement de notre analyse coté paramètres de soudage.
\end{abstract}

Mots clés : Éléments-finis / dureté / soudage / aluminothermie / défauts / porosité / adhésion

\begin{abstract}
Effects of alumino-thermic welding defects on the rails mechanical behaviour. The rail assembly is generally done through an aluminothermic welding process. This process involves the casting of solder between the two rail ends to be assembled. The quality of this assembly is highly dependent on conditions during the realization that are usually difficult to meet. Indeed, conventional techniques for the control of rails are often shown as the presence of welding defects in the junction. The observations suggest that these defects are mainly of two types: defects of adhesion (or bonding defects) and porosity defects (or multiple blisters defects). Each of these defects may constitute a privileged site of stress concentration. Depending on the level of these stresses, potential damage process may be developed and put off the rail. It therefore seems necessary to model the mechanical behavior of these welds with these defects in order to classify them by their criticality. In this study, we therefore modeled with the same load and with the same boundary conditions, the weld seam for each type of defect. The results are very conclusive and can well guide the deepening of our analysis side welding parameters.
\end{abstract}

Key words: Finite-elements / hardness / welding / aluminothermic / defects / porosity / adhesion

\section{Introduction}

Compte tenu de l'augmentation des vitesses et de la fréquence de circulation des trains et afin de réduire les vibrations, les jonctions des rails des chemins de fer sont conçues de manière à obtenir la continuité du chemin de

\footnotetext{
${ }^{a}$ Auteur pour correspondance : i.mouallif@gmail.com
}

roulement. En général le raccordement des rails est réalisé par les deux procédés suivants :

- soudage par résistance;

- soudage par aluminothermie.

Ce deuxième procédé est le plus répandu actuellement dans les chemins de fer. Cependant, il a été constaté que l'endommagement des rails se produit le plus souvent dans ces zones soudées, ce qui peut avoir de graves 


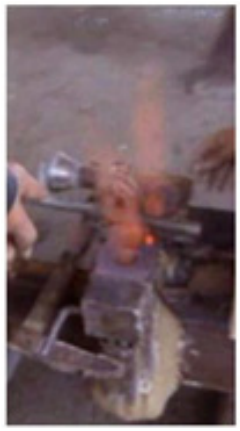

(a)

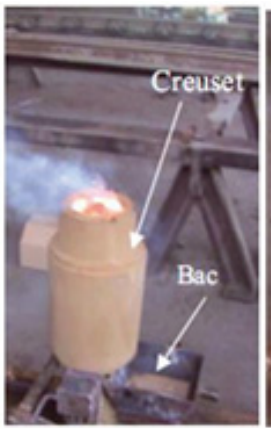

(b)

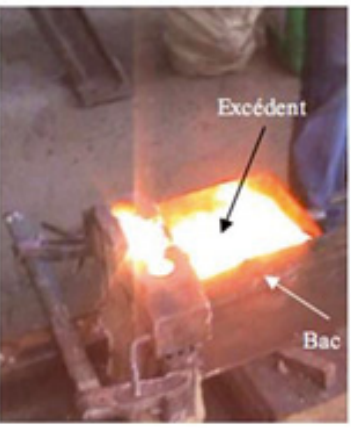

(c)

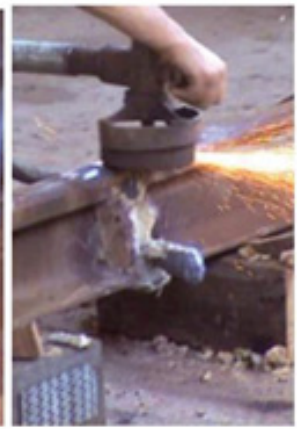

(d)

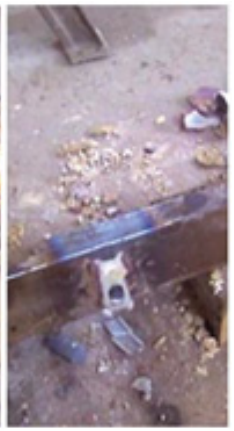

(e)

Fig. 1. Étapes d'exécution d'une soudure aluminothermique : (a) préchauffage, (b) déclenchement de la réaction aluminothermique, (c) solidification, (d) meulage et (e) finition.

conséquences sur la sécurité et le confort au cours du fonctionnement.

En effet, lors du déraillement du train 5941 entre Andelat et Saint Flour (France) le 25 février 2006, le Laboratoire Central des Ponts et Chaussées LCPC ainsi que le Centre d'expertise de la SNCF ont mis en cause : «...le défaut présenté comme amorce situé dans le métal d'apport en dehors de la zone de fusion du rail lui-même (zone de collage entre métal d'apport et rail)... la rupture du rail est liée à la fragilité du matériau et à la présence de points singuliers créés par la soudure par aluminothermie. La petite soufflure observée dans le métal fondu est l'une des particularités ayant pu amorcer la rupture fragile du rail...» $[1]$.

Dans le but de mieux cerner ce phénomène nous proposons dans cet article une modélisation d'une soudure aluminothermique contenant des défauts volumiques (défauts de porosité ou de soufflures multiples) et des défauts plans (défauts d'adhésion ou de collage).

Ces défauts que nous avons constatés à partir des échantillons des soudures rompues, sont positionnés dans la zone de soudage. Cette modélisation est faite en considérant des formes différentes. Une forme sphérique est proposée pour la porosité et le manque d'adhésion est représenté par un espace vide dans l'interface rail/soudure. Les contraintes ont été analysées au voisinage immédiat de ces défauts, les calculs sont faits en considérant différents cas de chargement, cela en utilisant la méthode des éléments-finis à l'aide du code de calcul ANSYS.

\section{Caractérisation de la soudure aluminothermique}

Au Maroc, l'assemblage des rails de chemin de fer se fait par le procédé de soudage aluminothermique avec préchauffage limité (PL), il est semblable au procédé de soudage SkV-F qui est plus utilisé en Angleterre et en Australie [2-4]. Ce procédé est caractérisé par un paramètre clé, c'est le temps de préchauffage.

Ce dernier est effectué pendant une durée bien déterminée (cinq minutes), sans contrôle de la température obtenue en fin de séquence, il est réalisé à l'aide d'équipement suivant : une bouteille de propane, un brûleur air et un mano-détendeur.

Les étapes de la réalisation de ce procédé sont décrites à travers la figure 1 .

\subsection{Propriétés mécaniques}

Pour les propriétés mécaniques, nous avons caractérisé la zone fondue à partir des essais de dureté. Les résultats obtenus sont donnés dans la figure 2 .

Cette figure montre l'évolution de la dureté Brinell sur une ligne dans la surface longitudinale du rail. Nous constatons que la dureté dans la zone fondue est supérieure à celle obtenue dans le métal de base (1 et 2). Cela est dû au refroidissement brutal de la soudure lors d'exécution du soudage aluminothermique. Ceci pourrait être assimilé à un phénomène de trempe générant un agrandissement des grains rendant la soudure fragile mais dure en surface.

À partir de ces résultats de l'essai de dureté, nous avons déduit pour à la zone fondue, un module de Young $E_{\mathrm{ZF}}=243 \mathrm{GPa}$ [5]. Le même ordre de grandeur a été obtenu expérimentalement pour certains aciers [6].

\section{Contrôle de la jonction de soudure}

Pour la sécurité des systèmes ferroviaires, le contrôle de la voie est réalisé périodiquement. Ce contrôle est assuré par un engin d'auscultation des rails (Fig. 3a), qui permet de donner un enregistrement automatique global. D'après cet enregistrement on peut savoir le type de défaut, sa position, sa densité et sa taille dans toute la ligne.

\subsection{Méthode de contrôle}

Les systèmes embarqués à traitement automatisé fournissent des informations qu'il est parfois nécessaire de confirmer ou d'affiner; pour cela des opérateurs se rendent sur la voie aux points kilométriques repérés par les voitures d'inspection, et mettent en œuvre des mesures 


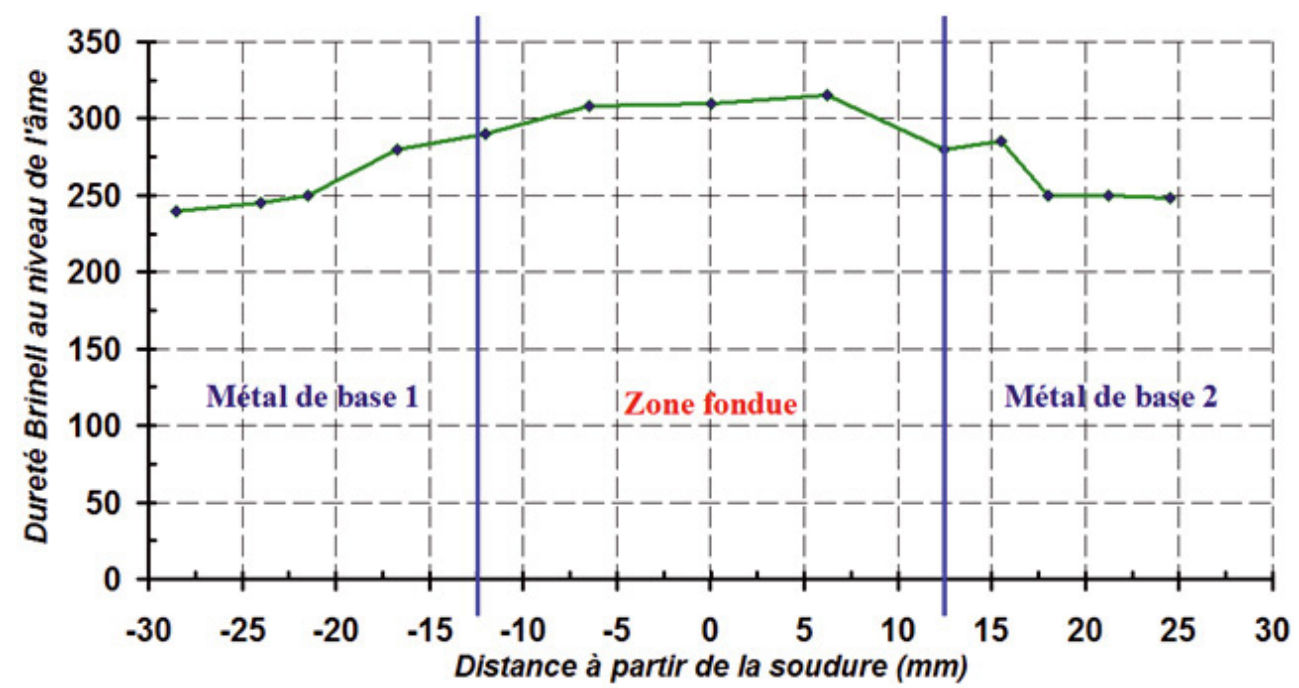

Fig. 2. Résultat d'essai de dureté Brinell selon la norme NF EN ISO 6506-1.

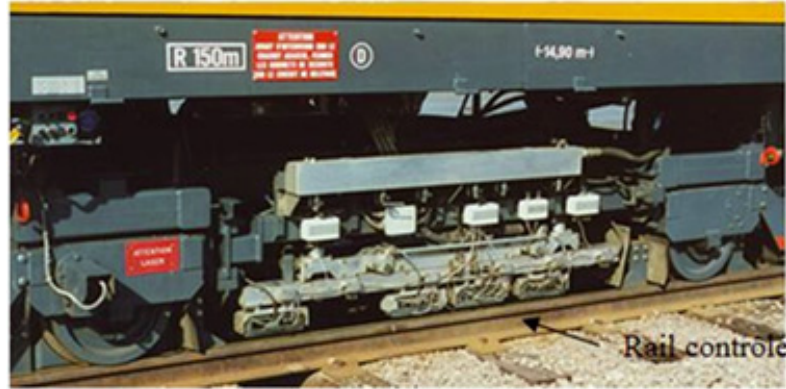

(a)

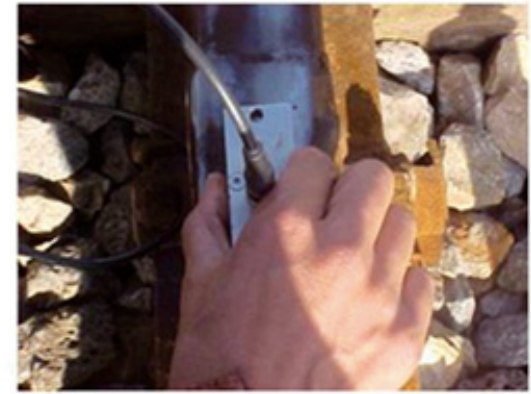

(b)

Fig. 3. (a) Contrôle global du rail, (b) contrôle local avec dispositif manuel.

complémentaires (Fig. 3b). Un examen ultrasonique manuel est réalisé avec des durées d'intervention plus grandes et des « angles » d'inspection plus variés, facilitant le diagnostic. Ces « confirmateurs » proposent alors un classement de gravité du défaut, allant d'une simple mise en observation à un retrait immédiat de la section de rail incriminée.

\subsection{Résultats et types de défauts}

Pour pouvoir identifier les différents défauts de rails, plusieurs sondes sont utilisées avec des orientations adaptées aux directions des anomalies recherchées (Fig. 4) et dans une gamme de fréquences allant de 1 à $10 \mathrm{MHz}$ [7] :

- un sondage oblique pour les défauts plans (cas d'adhésion) figure 4a;

- un sondage vertical pour le défaut volumique (cas de porosité) figure $4 \mathrm{~b}$.

À travers le contrôle des différentes jonctions de soudures, nous avons mis en évidence la présence de divers défauts. Ces défauts peuvent avoir des formes géométriques différentes (Fig. 3). Pour les mettre en évidence, nous avons réalisé des observations.

\section{Observation des types de défauts}

À partir des observations, nous avons pu classer les défauts générés par soudage aluminothermique suivant deux types : défaut d'adhésion (Fig. 5a) et de porosité (Fig. 5b).

\subsection{Défaut d'adhésion}

Le défaut d'adhésion (ou de collage) est un défaut majeur dans les chemins de fer marocains. Ce défaut génère $75 \%$ des ruptures transversales au niveau de l'interface rail/soudure (cela a été confirmé par l'ONCF) et qui pourrait être généré par une insuffisance en préchauffage.

\subsection{Défaut de porosité}

Au moment de l'exécution d'une soudure, l'emprisonnement de gaz lors du moulage génère un défaut sur le métal d'apport, qui s'appelle le défaut de porosité (ou soufflures multiples).

Les soudures affectées ont été caractérisées après rupture par une grande abondance de soufflures donnant au 


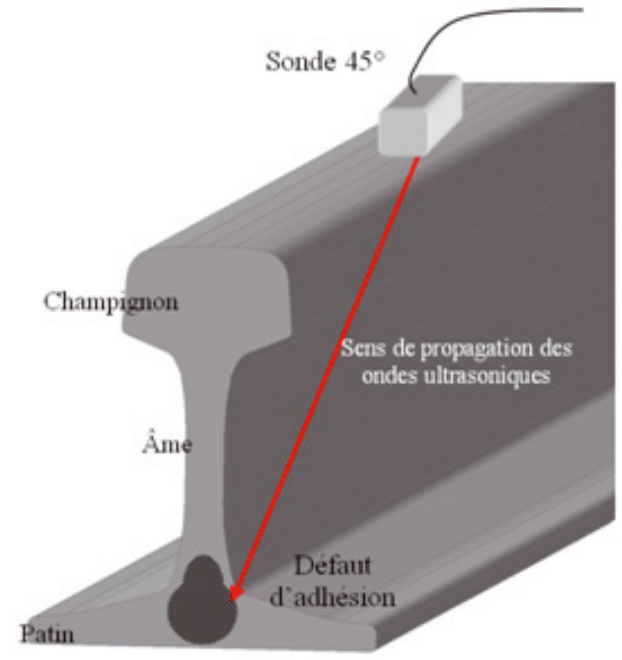

(a)

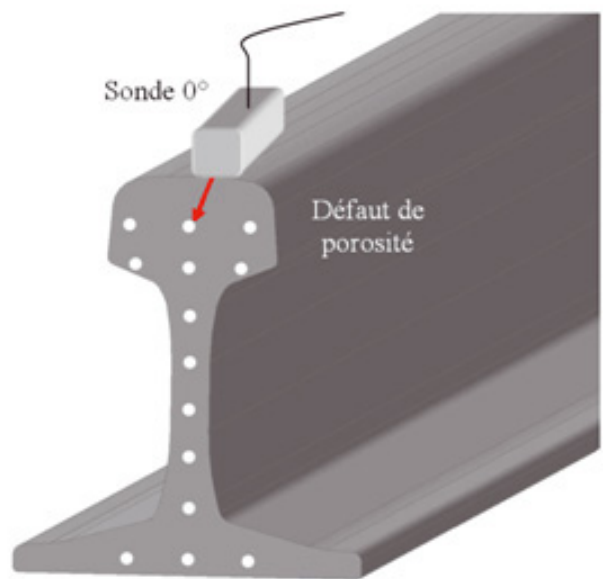

(b)

Fig. 4. Détection de la présence des défauts : (a) d'adhésion, (b) de porosité.

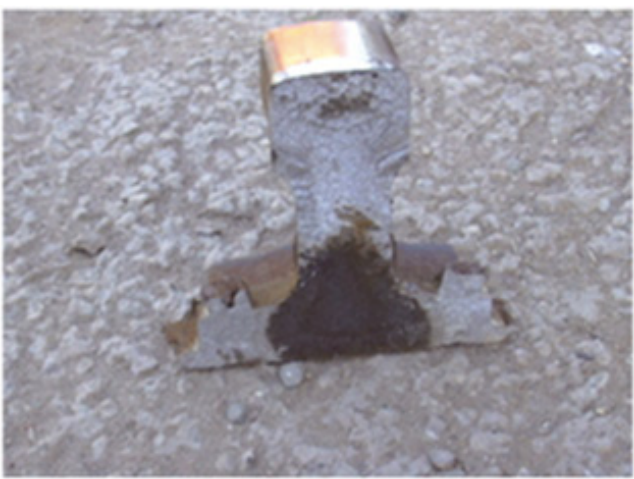

(a)

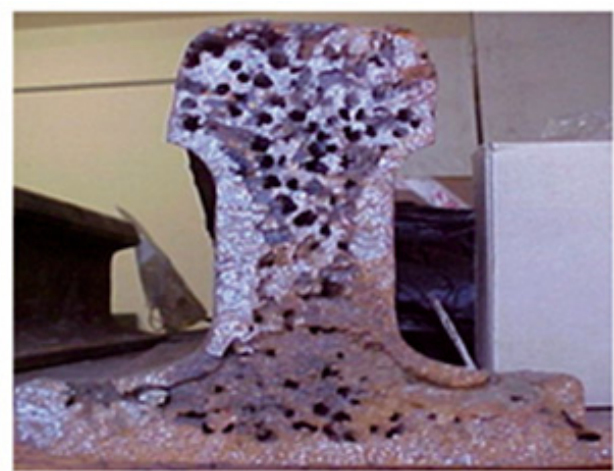

(b)

Fig. 5. (a) Défaut d'adhésion (ou défaut de collage), (b) défaut de porosité.

métal une allure « nid d'abeilles » à l'intérieur de la soudure (Fig. 5b).

Ce défaut résulte généralement de l'utilisation d'une charge humide ou d'une coulée dans un creuset ou un moule insuffisamment étuvé [8].

Parmi les facteurs qui pourraient être à l'origine de ce type de défaut on peut citer : insuffisance de préchauffage, changement climatique, erreurs d'exécution, présence d'humidité dans le moule, le creuset ou la charge aluminothermique.

Les différents défauts que nous avons identifiés précédemment favorisent manifestement des concentrations de contraintes. Selon le niveau de ces contraintes, des processus d'endommagement peuvent se développer particulièrement dans la zone de soudure ou affectée thermiquement (ZAT), comme cela a été confirmé par les statistiques de la figure 6 [9]. Par ces phénomènes, la durée de vie du rail se réduit. Il semble donc nécessaire de modéliser le comportement mécanique de ces jonctions en vue de distinguer ces défauts par leur sévérité et par conséquent corriger par priorité les paramètres de réalisation.

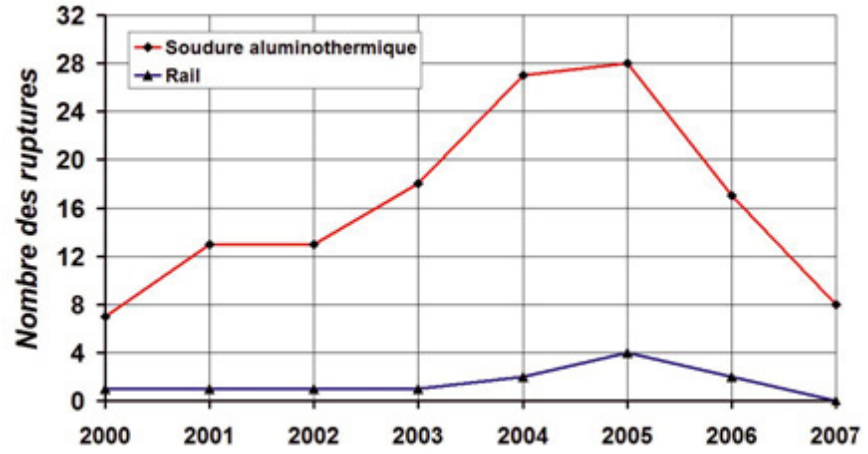

Fig. 6. Statistiques des ruptures des rails et des soudures aluminothermiques (2000-2007) sur une ligne de $81 \mathrm{~km}$ entre S Elaidi-Khouribga [9].

\section{Modélisation des défauts de soudure aluminothermique}

Dans cette partie, nous avons considéré une locomotive de 21,120 tonnes (2640 kg/roue). La surface de 


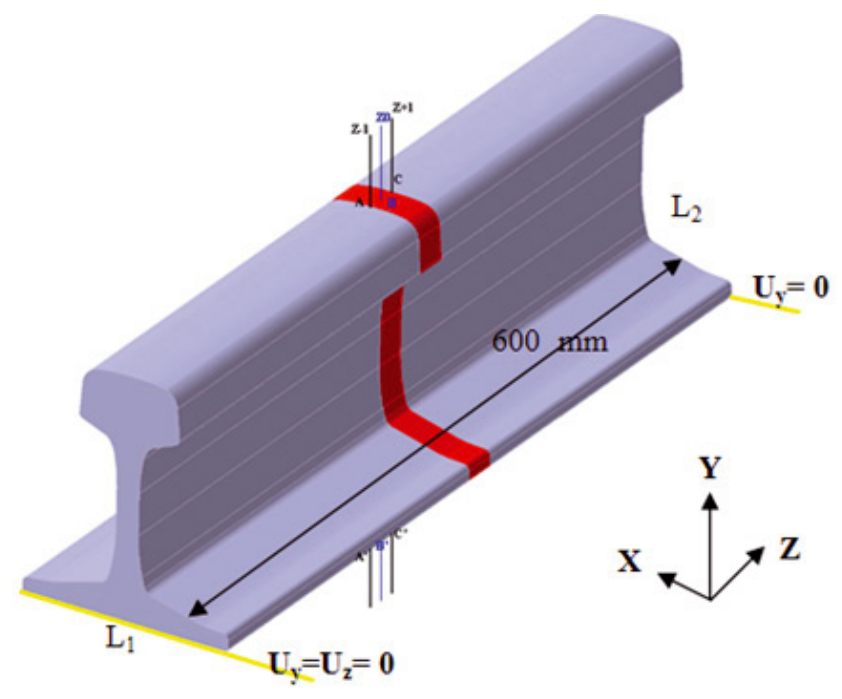

(a)

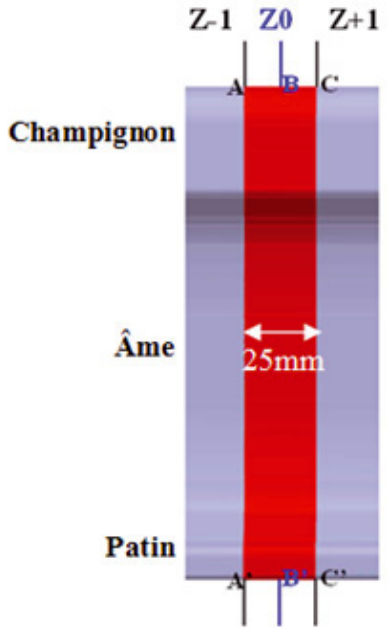

(b)

Fig. 7. (a) Modélisation géométrique et conditions aux limites d'une portion du rail comportant une jonction de soudure. (b) Repérage dans le plan $(Y, Z)$ des interfaces MB/ZF $(Z+1$ et $Z-1)$ par rapport à l'axe central $(Z 0)$ de la soudure.

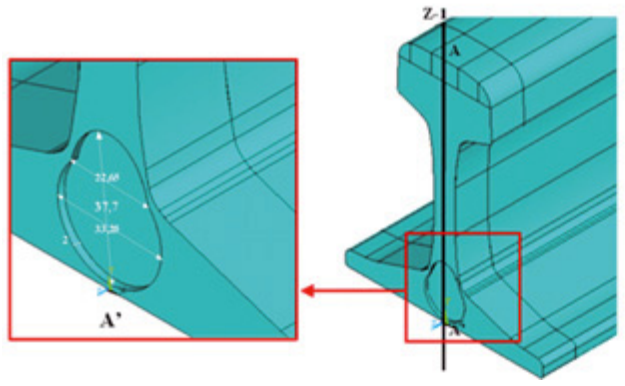

(a)

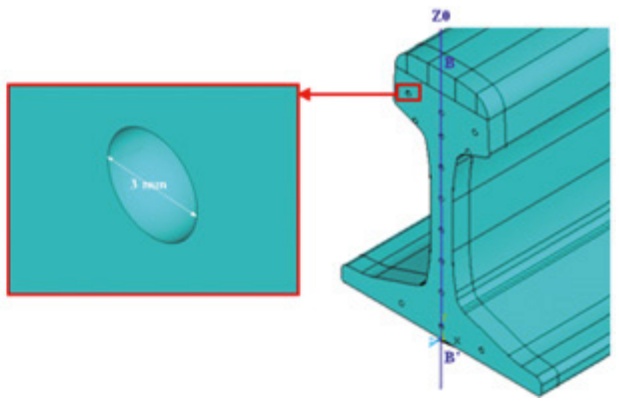

(b)

Fig. 8. (a) Modèles de défaut d'adhésion et (b) défaut de porosité.

contact $\left(S=51,26 \mathrm{~mm}^{2}\right)$ est modélisée par un contact hertzien. Comme nous l'avons déjà développé [10], la charge appliquée dans cette simulation est donc de l'ordre de $505 \mathrm{MPa}$.

Remarquons que l'ordre de grandeur de la surface du contact relativement petite, engendre l'importance des pressions exercées sur cette surface.

Le profil de rail Vignole que nous avons considéré est de type UIC 60 [11-13]. Ce dernier est défini par la norme NF A 45-317 [14].

À partir des observations des défauts, nous avons modélisé les différents cas suivants :

- défauts d'adhésion simulés par l'existence d'une forme quelconque (ne formant pas des angles aigus) se localisant à l'interface rail/soudure au niveau central du patin, (Fig. 8a) ;

- défauts de porosité simulés par l'existence d'une abondance de cavités sphériques $(D=3 \mathrm{~mm})$ dans la zone soudée (Fig. 8b).

Par la suite, nous avons considéré une pression hertzienne moyenne de $505 \mathrm{MPa}$.
À partir de cette modélisation nous avons donc simulé par la méthode des éléments-finis, le comportement mécanique du rail soumis à un chargement hertzien moyen de $505 \mathrm{MPa}$. En plus de ce chargement, nous avons tenu compte de la contrainte résiduelle thermomécanique par une variation de température $\left(\Delta T=T_{\text {réf }}-T_{\text {Fusion }}\right)$, avec $T_{\text {réf }}=20{ }^{\circ} \mathrm{C}$ (température de référence) et $T_{\text {Fusion }}=$ $600{ }^{\circ} \mathrm{C}$ (température de fusion).

Pour ce faire, nous avons considéré une portion du rail longue de $600 \mathrm{~mm}$ (Fig. 7a). Cette portion comporte la zone de soudage limitée entre la position $(Z-1)$ et $(Z+1)$ comme nous l'avons indiquée sur la (Fig. 7a). La largeur de cette zone est prise égale à $25 \mathrm{~mm}$ (Fig. 7b).

Les conditions aux limites que nous avons prises en compte sont : les déplacements suivant $Y$ et $Z$ sont nuls sur la ligne (L1) et seulement le déplacement suivant $Y$ est nul sur la ligne (L2) (Fig. 7a).

Dans cette modélisation nous avons considéré deux configurations associées aux deux défauts mentionnés cidessus.

Dans la première configuration, nous avons assimilé le défaut d'adhésion à un évidement de matière localisé dans la partie centrale du patin (Fig. 8a). La forme 


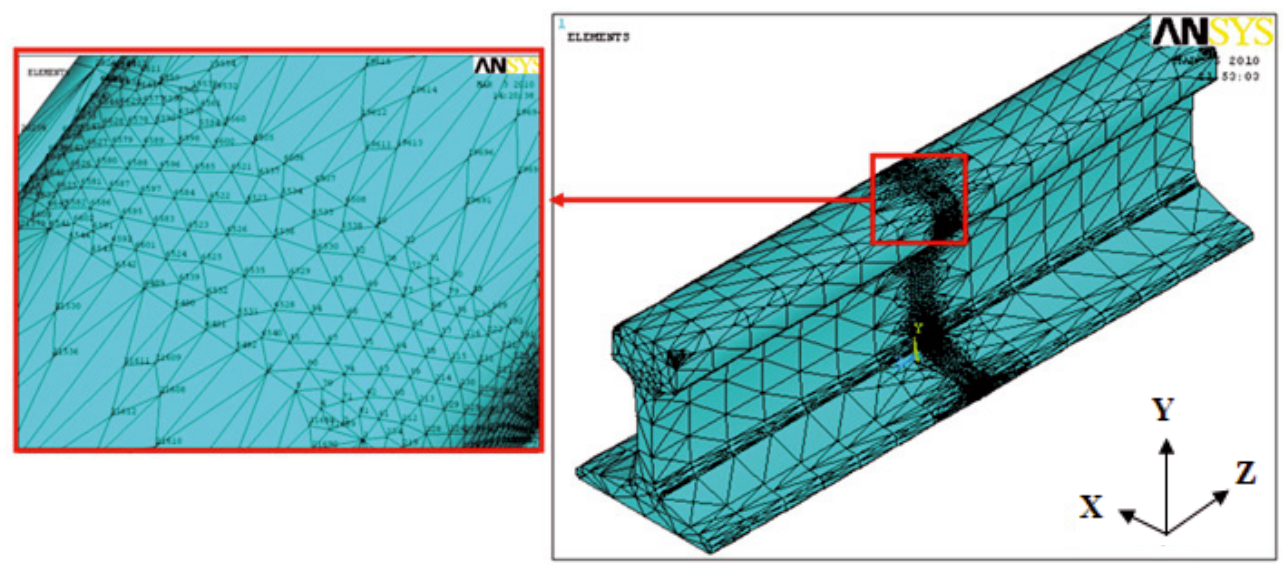

Fig. 9. Maillage de la structure étudiée par des éléments volumiques de type SOLID 185 (ANSYS).

géométrique de cet évidement a été définie à partir des observations. Dans la seconde configuration, le défaut de porosité, a été représenté par des cavités sphériques de diamètre $3 \mathrm{~mm}$, réparties dans tout le volume de la zone de soudure (Fig. 8b).

Les deux configurations ont été maillées avec des éléments volumiques de type SOLID 185 du code ANSYS (Fig. 9), pour le maillage de la structure et particulièrement au niveau de la jonction de soudure, jusqu'à ce que la solution en contrainte n'évolue plus en fonction de cette taille. Le nombre d'éléments considéré est de 711008 , dont $50 \%$ a été attribué à la zone de jonction de soudure. En effet, le raffinement du maillage au niveau de cette zone particulière nous permettra de développer notre analyse à partir des résultats précis. Le matériau considéré est l'acier avec comme module de Young $200 \mathrm{GPa}$ pour le rail et $243 \mathrm{GPa}$ pour la soudure. Le coefficient de Poisson est pris égal à 0,3 .

\section{Résultats et discussions}

A travers la simulation de la structure étudiée (portion du rail (Fig. 7), nous avons donc cherché à mettre en évidence le champ de contrainte développé à partir d'une charge moyenne.

Compte tenu de ce chargement et des conditions aux limites considérées, le comportement de cette portion du rail est celui d'une poutre en flexion dans le plan $(Y$, $Z)$. Cette flexion génère une contrainte de flexion $S_{Z Z}$, une contrainte de compression $S_{Y Y}$ et une contrainte de cisaillement due à l'effort tranchant $S_{Y Z}$. Ces trois composantes de contrainte sont mises en évidence à travers la figure 10 .

Pour analyser ces contraintes, nous allons distinguer trois cas de figures. Le premier cas est celui de la structure sans défaut. Le deuxième et le troisième cas correspondent aux structures comportant respectivement le défaut d'adhésion et le défaut de porosité.

Pour le premier cas (sans défaut), les résultats que nous avons obtenus sont illustrés à travers les graphiques de la figure 11. À partir de ces graphiques, nous pouvons constater que la contrainte de flexion $S_{Z Z}$ est en compression ( $\sim-140 \mathrm{MPa})$ au niveau de la surface libre du champignon et elle est en tension $(\sim 100 \mathrm{MPa})$ au niveau du patin. Entre ces deux valeurs extrêmes, l'évolution de la contrainte n'est pas linéaire compte tenu de la forme particulière de la section de la structure étudiée représentée dans l'arrière plan de chaque graphique. En revanche, les autres composantes $S_{Y Y}$ et $S_{Y Z}$ s'annulent en surface libre et ne dépassent pas la valeur $60 \mathrm{MPa}$ pour $S_{Y Y}$. Par rapport à la contrainte de flexion $S_{Z Z}$, la contrainte de cisaillement $S_{Y Z}$ est négligeable, comme on a l'habitude de le voir pour les poutres classiques.

Pour les cas des structures avec défauts, nous avons regroupé les résultats dans la figure 12. Selon ces résultats, nous pouvons constater que le défaut d'adhésion a généré localement une perturbation des champs de contrainte. En effet, au niveau des bords de ce défaut, des pics ont été développés pour toutes les composantes. La valeur maximale de ces pics a été obtenue pour la composante $S_{Z Z}$ à proximité de la surface libre du patin et elle est de l'ordre de $290 \mathrm{MPa}$.

Pour le défaut de porosité, les perturbations du champ de contrainte n'ont pas été significatives. Seule l'évolution de la composante $S_{Y Y}$ a été caractérisée par des petits pics localisés et dont le niveau reste globalement faible (Fig. 13).

\section{Conclusion}

À travers des observations des jonctions de soudure, nous avons pu caractériser les différents défauts générés lors de la réalisation. Parmi ces défauts nous avons retenu pour les modélisations par éléments-finis, les défauts de porosité et d'adhésion qui sont très fréquents. Dans ces modélisations nous nous sommes basés sur des configurations géométriques extraites des observations.

Les premiers résultats des modélisations que nous avons obtenus montrent clairement que ce sont les défauts d'adhésion qui ont tendance à concentrer le plus de contraintes. De plus, ils sont généralement situés au niveau des interfaces rail/soudure, zone privilégiée pour 


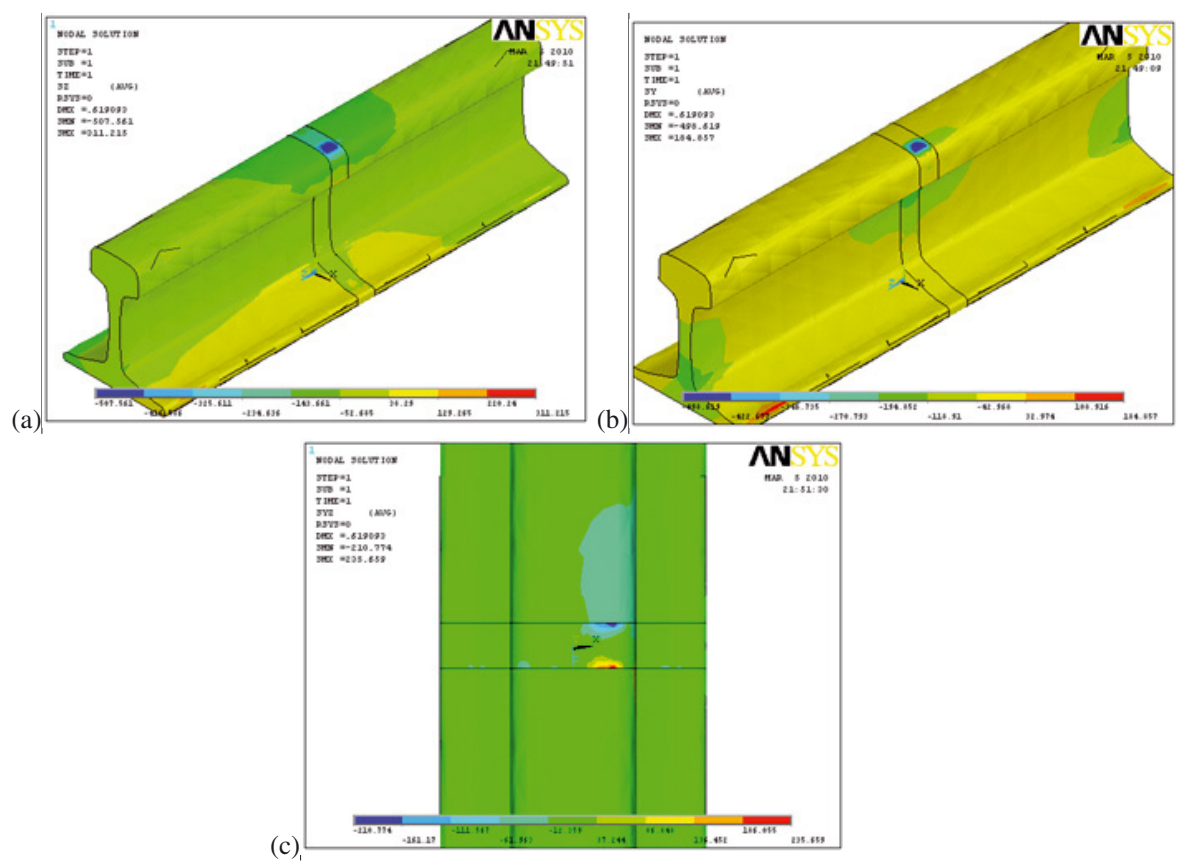

Fig. 10. Distribution des contraintes pour le cas de défaut de porosité (a) contrainte de flexion $S_{Z Z}$, (b) contrainte de compression $S_{Y Y}$ et (c) contrainte de cisaillement $S_{Y Z}$.

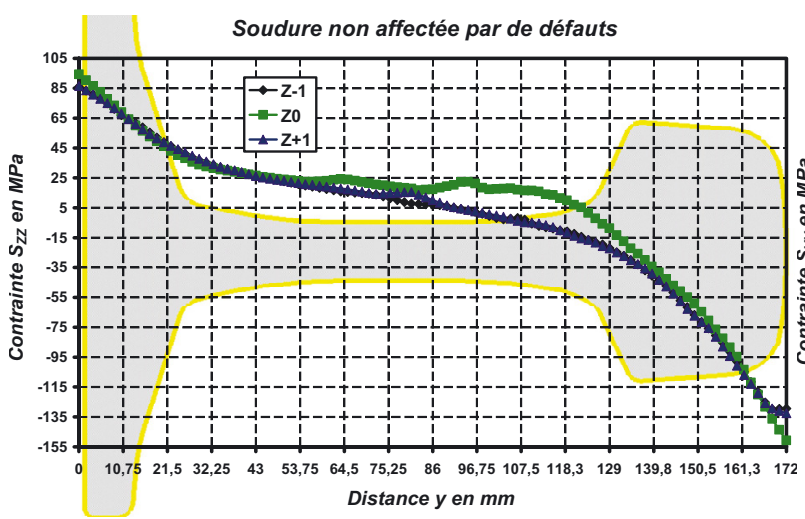

(a)

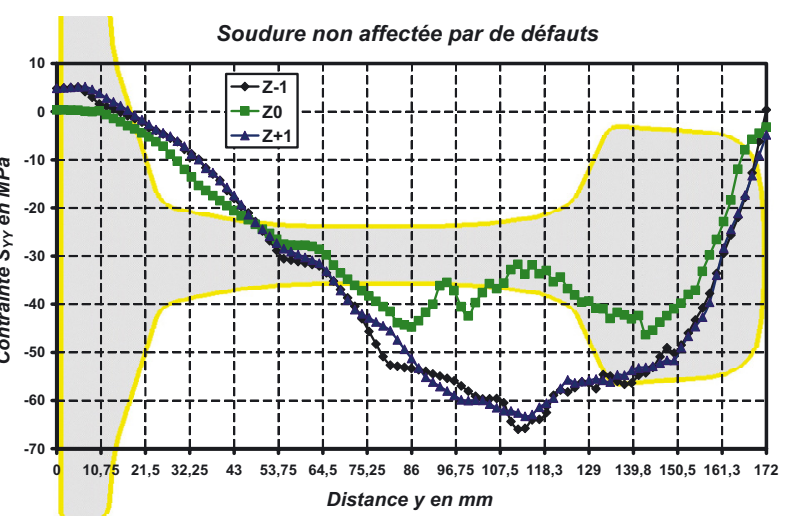

(b)

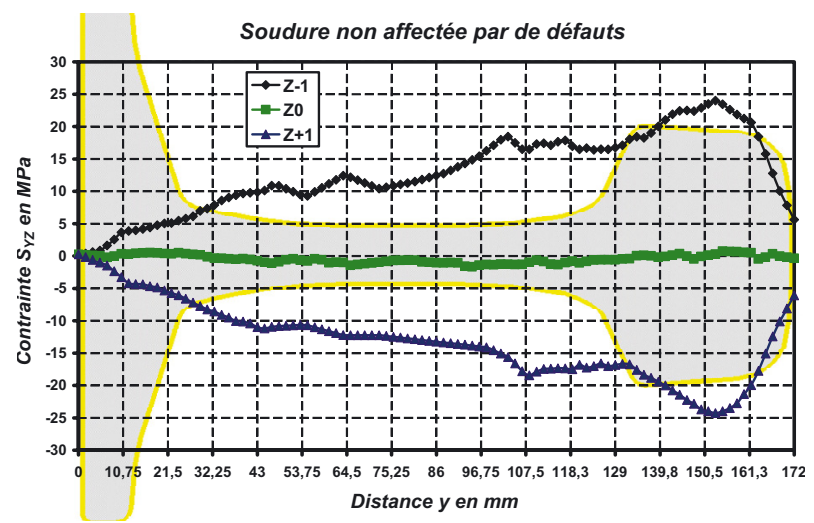

(c)

Fig. 11. (a) Distribution de contraintes de flexion $S_{Z Z}$ en fonction de la distance $y$, pour une soudure non affectée par les défauts. (b) Distribution de contraintes de compression $S_{Y Y}$ en fonction de la distance $y$, pour une soudure non affectée par les défauts. (c) Distribution de contraintes de cisaillement $S_{Y Z}$ en fonction de la distance $y$, pour une soudure non affectée par les défauts. 


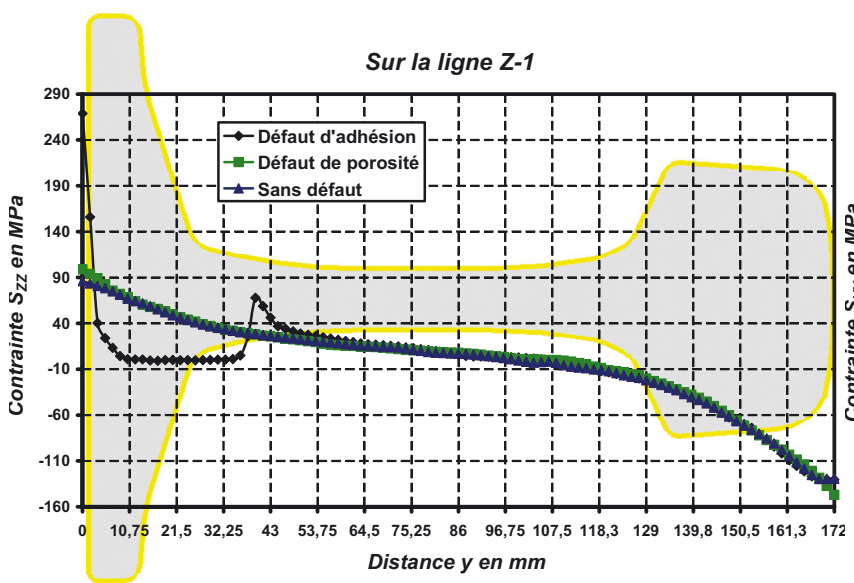

(a)

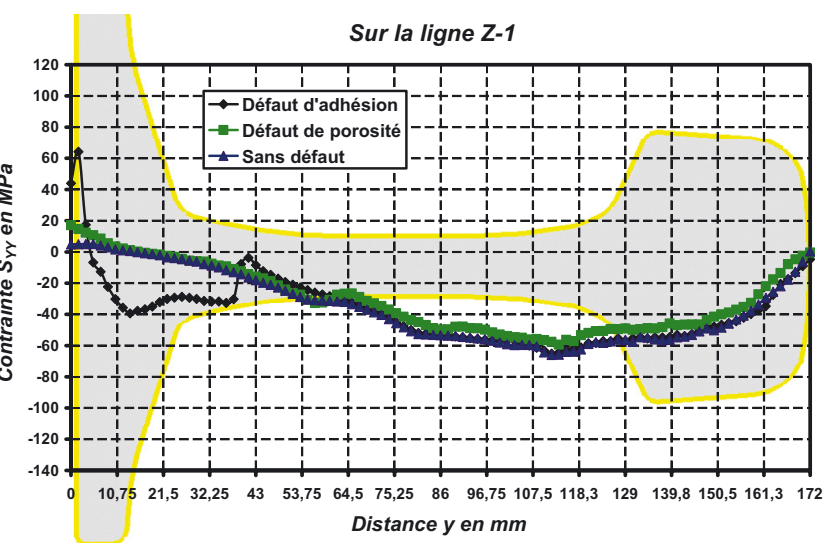

(b)

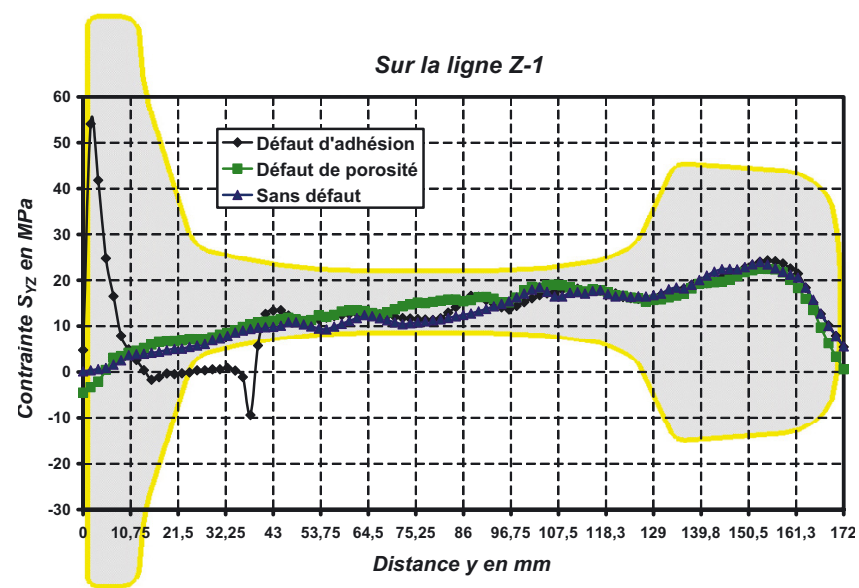

(c)

Fig. 12. (a) Distribution de contraintes de flexion $S_{Z Z}$ en fonction de la distance $y$, le long de la ligne verticale $Z-1$. (b) Distribution de contraintes de compression $S_{Y Y}$ en fonction de la distance $y$, le long de la ligne verticale $Z-1$. (c) Distribution de contraintes de cisaillement $S_{Y Z}$ en fonction de la distance $y$, le long de la ligne verticale $Z-1$.

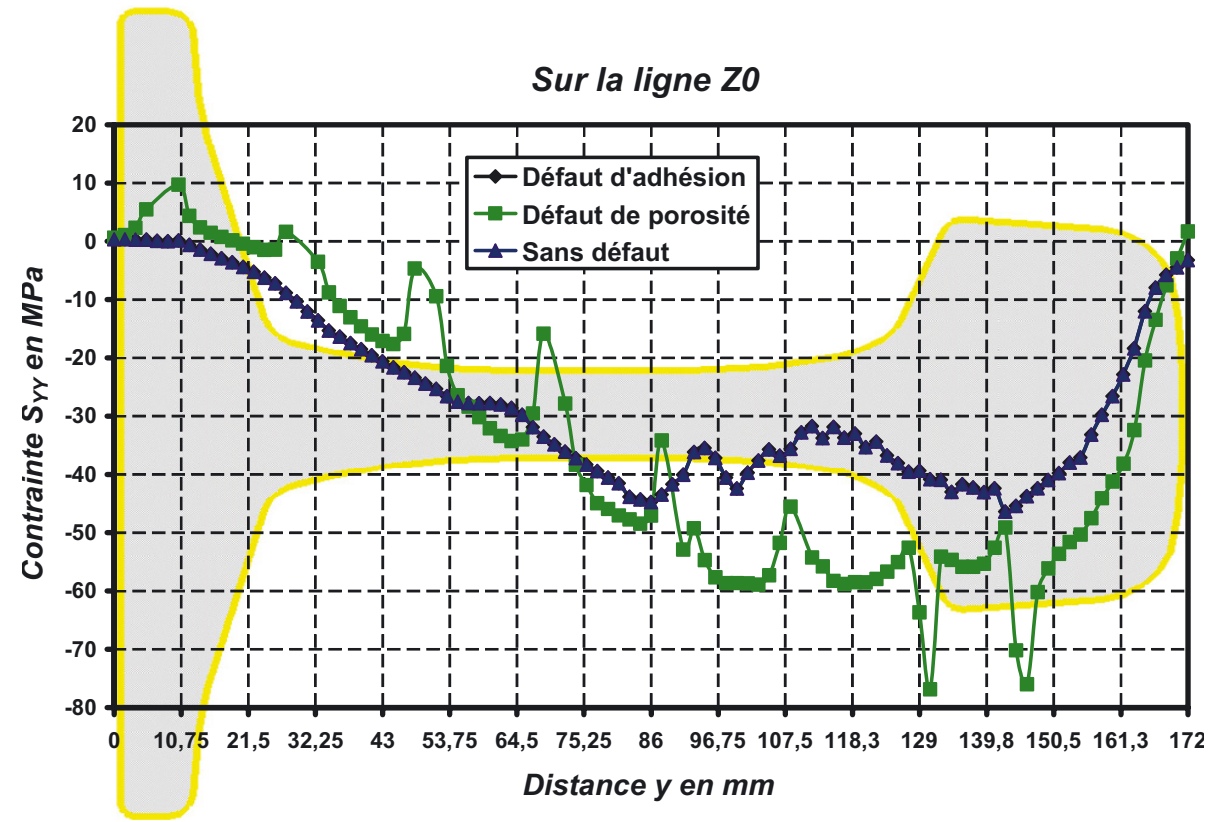

Fig. 13. Distribution de la contrainte de compression $S_{Y Y}$ en fonction de la distance $y$, le long de la ligne verticale $Z 0$. 
des éventuels processus d'endommagement même si on a localement la relaxation des contraintes résiduelles. La caractérisation de cet endommagement nécessite une analyse asymptotique permettant la détermination des grandeurs qui gouvernent la propagation de fissure à partir de ces défauts.

Ces défauts particuliers qui retiennent notre attention pour approfondir nos modélisations, semblent provenir d'un mauvais préchauffage. En effet, ces défauts observés au niveau central du patin se présentent sous forme de tache noire (excès de carbone). Celle-ci s'expliquerait par un manque d'oxygène lors de préchauffage qui est toujours localisé dans des zones éloignées à la source de préchauffage. Ce manque d'oxygène provoquerait une réaction de combustion locale non parfaite, par conséquent la température locale dans cette zone n'atteindrait pas la valeur voulue.

Donc la suite de cette étude comportera une analyse thermique à partir de laquelle on simulera ces mauvaises conditions de préchauffage et mettra en évidence son effet sur la description locale du champ de contraintes. D'autre part, il nous semble indispensable de développer cette analyse à partir d'un chargement de fatigue.

\section{Références}

[1] Bureau d'enquêtes sur les accidents de transport terrestre, BEA-TT, Rapport d'enquête technique sur le déraillement du train 5941 survenu le 25 février 2006 à Saint Flour (15), pp. 20-21

[2] P.-J. Mutton, E.-F. Alvarez, Failure modes in aluminothermic rail welds under high axle load conditions, Eng. Fail. Anal. 11 (2004) 151-166

[3] R. Moller, P. Mutton, M. Steinhorst, Improving the performance of aluminothermic rail welding technology, through selective alloying of the rail head, Proc. 7th International Heavy Haul Conference, Brisbane, 2001, IHHA, pp. 331-338
[4] Thermit Welding, Thermit Australia Pty Ltd, The SkV-F process

[5] I.M. Meththananda, S. Parker, M.P. Patel, M. Braden, The relationship between Shore hardness of elastomeric dental materials and Young's modulus, Dent. Mater. 25 (2009) 956-959

[6] L. Avril, Elaboration de revêtements sur acier inoxydable : simulation de la fusion par irradiation laser caractérisation structurale, mécanique et tribologique, J.-J. Hantzpergue, B. Courant, Angers, 2003

[7] Matix Industries, auscultation des rails par ultrasons, document technique, 1984

[8] J.-C. Alacoque, P. Chapas, Transport ferroviaire : gestion de l'adhérence, pp. 6-8

[9] Direction Maintenance Infrastructure de l'ONCF, Étude statistique des défauts de rails et de soudures aluminothermiques sur LRS, document technique, 2007

[10] I. Mouallif, A. Chouaf, A. Elamri, A. Benali, Étude du comportement mécanique des rails soudés par aluminothermie, Communication aux VI Journées d'Études Techniques 2010, Marrakech, Maroc, 2010

[11] I. Mouallif, A. Elamri, A. Chouaf, A. Benali, Étude du comportement des rails soudés par aluminothermie, Communication au $3^{\text {e }}$ Congrès International Conception et Modélisation des Systèmes Mécaniques CMSM'2009, Hammamet, Tunisie, 2009

[12] I. Mouallif, A. Elamri, A. Chouaf, A. Benali, Étude du comportement mécanique des rails soudés par aluminothermie, Communication au $9^{\mathrm{e}}$ Congrès de Mécanique, Marrakech, Maroc, 2009

[13] I. Mouallif, A. Benali, A. Elamri, A. Chouaf, Étude du comportement des rails soudés par aluminothermie, Congrès Algérien de Mécanique CAM2009, Biskra, Algérie, 2009

[14] NF A 45-317 Produits sidérurgiques laminés à chaud, Rail Vignole type $60 \mathrm{~kg} / \mathrm{m}$ et éclisse, Profil : caractéristiques et tolérance 